\title{
Review
}

\section{Peyronie's Disease: Management and New Therapeutic Acquisitions}

\author{
Alain Bitton*, Marco Firmo, and Bruno Boccioli \\ Urological Surgeon, Andrology and Men's Health Clinic, Switerland
}

\begin{abstract}
${ }^{*}$ Correspondence to: Alain Bitton; Men’s Health and Andrology Clinic - 95 Route de Florissant - 1206 Genève - Médecin independent, Switerland; E-mail: drbitton@andrologue.com
\end{abstract}

Received: Nov 20 $0^{\text {th }}, 2020$; Revised: Nov 22 ${ }^{\text {nd }}, 2020$; Accepted: Nov 23 $3^{\text {th }}, 2020$; Published: Nov $24^{\text {th }}, 2020$

Citation: Bitton A, Firmo M, Boccioli B. Peyronie’s disease: Management and new therapeutic acquisitions. Urology Open A Open J. 2020; I(1): 15-30.

\begin{abstract}
Peyronie's disease is a condition known for a long time but which has long been stigmatized as a malformation, misunderstood and generating shame and embarrassment on the part of patients. Because of its multifactorial aspect and its association with certain metabolic diseases, the treatment for many years consisted of a simple observation, the prescription of vitamin E or other anti-inflammatory or vasodilator molecules. We present here a new acquisition thanks to the application of linear shock waves, a promising and quite interesting element in the therapeutic arsenal. This technique, especially when coupled with the use of PDE-5 inhibitors and electroporation should make it possible to treat and improve not only punctually the fibrous nodule by improving the elasticity of the tissues concerned, but also of activate the circulation of the cavernous bodies of the penis thus improving erectile function allowing to give hope to many patients who are desperately seeking a solution to their problem.
\end{abstract}

Keywords: Peyronie’s disease; Fibrous nodule; Multifactorial aspect; Vitamin E; New acquisition; Application of linear shock waves; IPP; PDE-5 inhibitors; Electroporation; Improving erectile function.

\section{PEYRONIE'S DISEASE}

\section{Description \& Diagnosis}

First described in 1743 by Louis XVI's famous surgeon François de La Peyronie, it is usually an induration of the corpora cavernosa associated with pain and penile curvature on erection. ${ }^{1}$ Unlike other male sexual pathologies, for which patients are slow to consult, in this debilitating pathology, which usually begins with a painful fibrous nodule, patients seek specialist advice fairly quickly.[Figure 1]

The incidence of the disease is more marked between 50 and 65 years, but can also appear at a young age and sometimes even as early as adolescence, especially in its congenital form. ${ }^{2}$ This pathology affects around $8 \%$ of the male population. ${ }^{3}$ The precise cause is unknown, probably of multifactorial origin. Several theories have been put forward, including the one implicating vascular microtrauma in albuginea during intercourse. Indeed, it would seem that in certain genetically predisposed subjects, there is an alteration of the repair processes
Figure 1. Relatively severe form of Peyronie's disease with extreme ventral curvature of more than $90^{\circ}$.

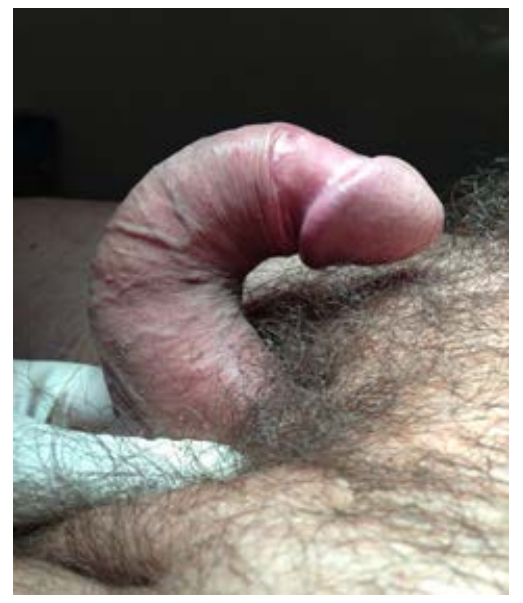


leading to an excessive production of TGF- $B 1$ (cytokine leading to the formation and maintenance of fibrosis of the cavernous bodies). It would therefore be an altered inflammatory response to trauma or repetitive strain injuries in genetically predisposed subjects. ${ }^{4,5}$

\section{Patient Approach}

In the initial phase it is obviously imperative to reassure the patient by an empathetic discussion as well as to confirm the diagnosis. The history will look for any previous trauma, even minimal, through "coitus missteps" and sexual intercourse in extreme positions. The use of certain rings to increase erection can also create fibrosis of the corpora cavernosa, due to repetitive strain injuries. Metabolic diseases that may be associated with Peyronie's disease include familial diabetes after the age of 50, hyperuricemia, hypertension and atherosclerosis. ${ }^{6,7}[$ Figure 2]

Figure 2. The fundamental element of the disease is the appearance, on the tunica albuginea (the sheath), of the cavernous bodies of the penis of a zone of hardening ("the plate") varying from a few $\mathrm{mm}$.

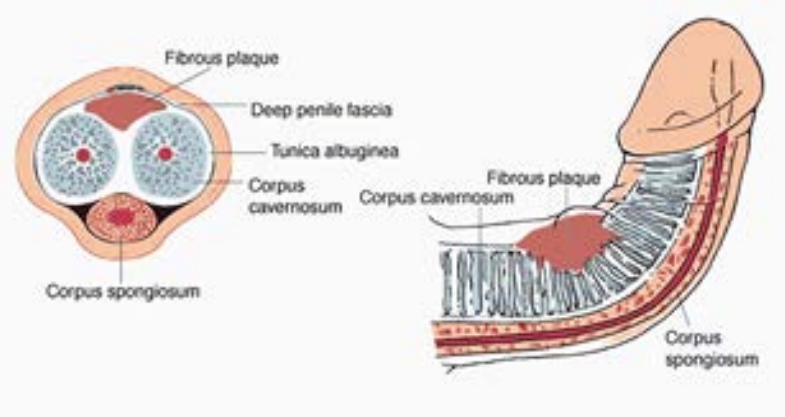

After the history, the specialist will perform a careful physical examination by palpating and measuring the points of fibrosis in the penis without forgetting the septum. The rest of the examination will also include looking for any abnormalities in the prostate, urology or even general. In fact, we sometimes find associated with this urological trophicity disease, fibrosis of the tendons of the hand known as Dupuytren's disease. ${ }^{8}$ It is interesting to note that in both pathologies, the disease begins with vasculitis, followed by exudation of plama and insufficient fibrinolysis which leads to the formation of plaque which over time can ossify.

\section{Additional Tests}

Ultrasound and Doppler echo with or without injection of vasoactive substances (prostaglandin or papaverine), will above all make it possible to evaluate the intensity of the plaque and the extent of the curvature with measurement of the angle, subsequently orienting management and allowing follow-up and a prognostic factor depending on the treatment chosen.

In rare cases, a penile MRI may be requested to look for tumor pathology (penile metastasis from prostate cancer, a rare but classic occurrence) or for a hematological disease which usually causes priapism in addition to the curvature and inflammatory nodule.
Figure 3. Ultrasound showing a plaque of circumscribed fibrosis of the tunica albuginea as well as the poorly vascularized sheath which covers the cavernous bodies of the penis. The area of fibrosis, generally defined as "plaque", is a limitation to the elasticity of the penis during erection causing a curvature towards the affected side.

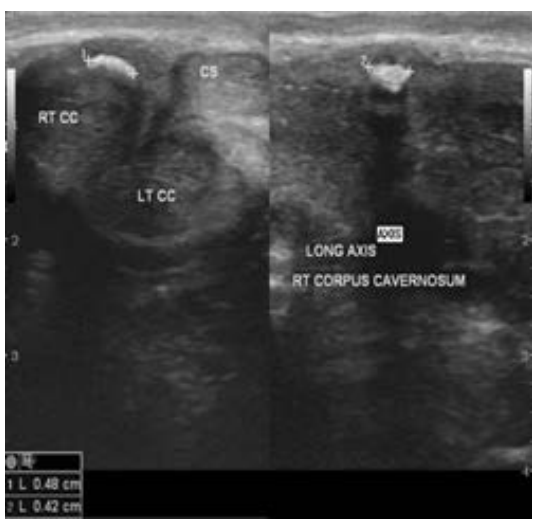

\section{Drug and Preservative Treatments}

For many years, treatments were confined to the prescription of vitamin E known for its antioxidant properties, as well as certain drugs to reduce the pain and inflammation of the plaque such as Veraparmil, hydrocortisone, Potaba (Aminobenzoic Acid), collagenase, hyaluronic acid, and certain other vasodilators. ${ }^{10,11}$

Some studies report therapeutic effects with interferon or intracavernous or transdermal applications of verapamil or collagenase. ${ }^{12-15}$ The results, although promising, are still insufficient to be considered as gold standards and to be applied routinely in the treatment of the disease.

\section{Non-drug Approaches}

In extreme cases such as in the congenital form or when drug treatments prove insufficient (difficulty or pain during erections or intercourse), the treatment offered was surgical. It should be mentioned, however, that in all cases, it is preferable to consider surgery between 9 months and two years after the onset of the disease due to the progressive aspect of the pathology and the spontaneous regression of the plaques and fibrosis. ${ }^{16}$ Indeed, even if one obtains a stabilization of the fibrotic process in 60 to $80 \%$ of the cases and an analgesic improvement in nearly $80 \%$ of the situations thanks to the medicinal treatments, the real problem for many patients is the persistence of a unsightly penile curvature with correction and partial regression of the plaque in only $20 \%$ of cases.

Apart from drugs, we must also mention the devices allowing regular penile extension or the vacuum pump (Vacuum) improve the elasticity of the tissues and decrease the long-term inflammatory process without really completely curing the fibrous process.

\section{Surgical Techniques}

Classically, the plication of the cavernous bodies according to the NESBIT technique with or without excision of fibrous tissue depending on the severity of the fibrosis, has been and remains the surgical "gold standard" of the pathology. ${ }^{17}$ Some of these kinks are simple, non-invasive and do not affect erectile capacity in any way. They are indicated for mild curvatures $\left(<30^{\circ}\right)$ without hypoplasia of the penis. ${ }^{17}$ However, due to penile straightening, however small, some "shortening" of the pe- 
nis is to be expected, proportional to the extent of flexion. The patient should be warned. ${ }^{18}$

In more severe situations, more complex and invasive techniques will be used, involving resections of large fibrous tissue with prosthetic replacement (grafts) of the excised tissue. As mentioned, it will be necessary to wait, especially for these surgical corrections, that the plaque is perfectly stabilized without new inflammatory outbreak. The replacement material used must meet certain requirements such as the prevention of penile retraction, the maintenance of good quality elasticity as well as histocompatibility in the case of grafts. ${ }^{19}$ When the surgery is well done, functional results are good with penile lengthening allowing sexual intercourse through sufficient penetration. ${ }^{20}$ Surgery with graft after excision of fibrous plaques can also be associated with the placement of a penile prosthesis in cases of erectile dysfunction and extensive intracavernous fibrosis with severe shortening of the penis. ${ }^{21}$

\section{NEW THERAPEUTIC ACQUISITIONS}

\section{Shock Waves}

Shock waves are mechanical waves similar to acoustic waves that can propagate in a medium. When these waves are applied to an organ or tissue, they release energy, causing compression and then expansion of the tissues encountered. It is the principle of "cavitation" that physicists are familiar with. The application of the mechanical wave at low intensity to the endothelium of blood vessels results in the formation of biochemical signals encouraging the formation of new small capillaries through growth factors: principle of neoangiogenesis. When the wave is applied to the cavernous bodies, it allows this phenomenon of neoangiogenesis to provide better permeability as well as an increase in blood circulation to the erectile tissues, thus resulting in better penile functioning. This treatment can be combined either with the electroporation that we will see in the next paragraph or with the regular and low dose intake of 5 phospodiesterase (PDE-5) inhibitors, such as Tadalafil, Vardenafil or Sildenafil.

Figure 4. The application of linear shock waves of low intensity to the cavernous bodies, allows the regeneration of erectile tissues through the principle of neoangiogenesis, thus improving the function of the penis.

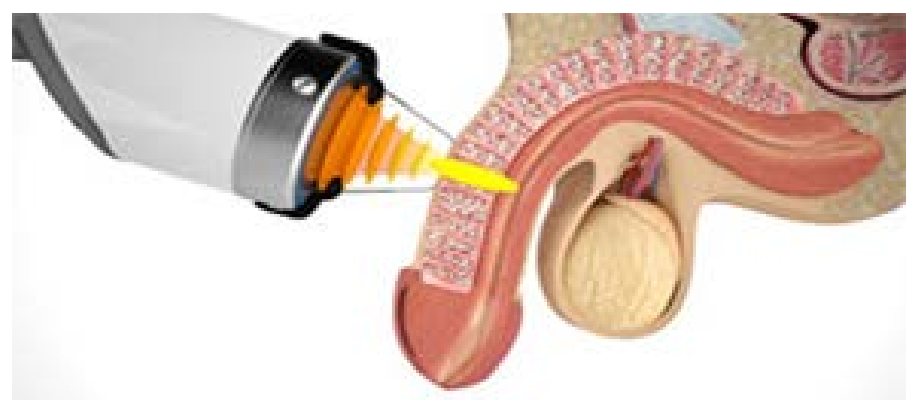

Table 1. The vascular response to shock waves is of two types.

\begin{tabular}{|l|l|}
\hline $\begin{array}{l}\text { Immediate response: } \\
\text { Transient vasoplegia }\end{array}$ & $\begin{array}{l}\text { Due to a temporary sympathetic effect induced by shock } \\
\text { waves on sympathetic nerve endings. It consists of local } \\
\text { vasodilation secondary to the release of pre-capillaries, due } \\
\text { to changes in cell permeability induced by shock waves. }\end{array}$ \\
\hline $\begin{array}{l}\text { Late and permanent } \\
\text { response }\end{array}$ & $\begin{array}{l}\text { The first mechanism follows for a few days and mainly cor- } \\
\text { responds to the process of neoangiogenesis. The formation } \\
\text { of new small vessels is the result of the release of a peptide } \\
\text { under the action of waves, causing an activation of type I } \\
\text { collagen. }\end{array}$ \\
\hline
\end{tabular}

\section{IPP Combi}

The IPP Combi is a device based on the principle of using low intensity mechanical waves applied to the penis. Although known and developed several years ago, the application of linear waves finds in the IPP Combi an interesting and completely original approach. The device was developed by an engineer, Professor Bruno Boccioli and developed for clinical application by a urologist-andrologist, Professor Marco Firmo. Our experience in Geneva is inspired by their work and their experience following a well-conducted education and training. The technique is used widely in Europe and the USA and has been introduced in Switzerland more than two years ago with increasing use. Treatment is outpatient and a series of treatment usually involves 6 applications, once or twice a week.

The treatment is simple and is performed on a patient either lying down or in a gynecological position allowing better access to the external genitalia, especially if one decides to treat the points of the perineum or the prostate during the same session. It is important that the patient is comfortable and well settled. The application of hard waves for 10 minutes at low intensity and medium frequency to be adapted according to the sensitivity of the patient and the quality of the tissues. The probe is applied directly to the cavernous body, starting the session away from the nodule and, if possible, in healthy tissue to avoid pain when applying energy to the sensitive area to be treated. The energy is triggered after switching on the device and adjusting the frequency and intensity by regular application on a foot control. The treatment is simple, painless and non-invasive and can be stopped at any time in order to adapt the constants according to the feelings and the sensitivity of the patient. After a few sessions, the tissues develop a certain memory of the energy applied and the treatment appears less sensitive with a better tolerance threshold. The protocol includes one to two sessions per week over a period of 6 weeks to be repeated after a few months depending on the progress. Usually after 3 to 4 sessions, patients note better spontaneous erections with softening of the nodule, reduced doses of PDE5 inhibitors. Improvement or straightening of the penile curvature, on the other hand, takes longer, ranging from 6 weeks to 6 months.[Figure 5]

Figure 5. Computerized treatment console including adjustable parametric constants for each patient according to the pathology to be treated.

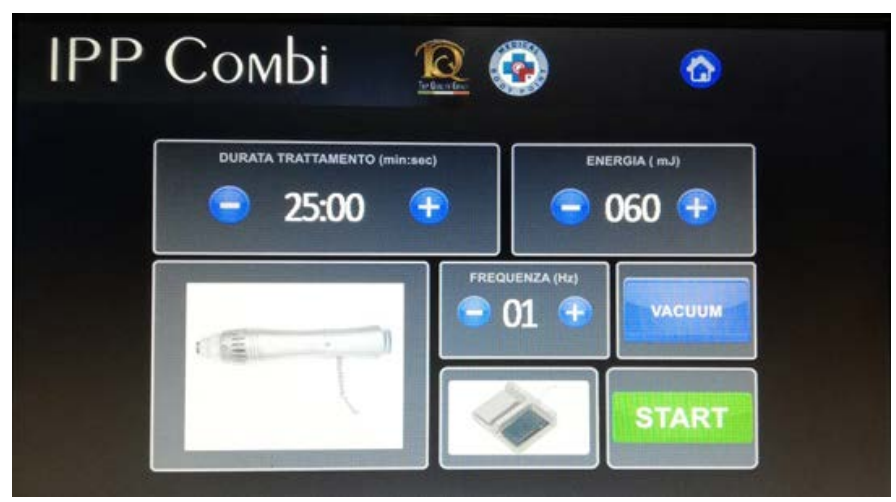

\section{Electroporation}

It is a non-invasive technique most often coupled with the application 
of mechanical waves, which involves applying an electric current to a surface such as the skin or a cell membrane. This process is used to open pores or channels through which a substance can pass. This method causes a reversible and momentary increase in tissue permeability by acting on the phospholipid component of cell membranes.[Figure 6,7,8] [Table 2]

Figure 6. Description of the principle of electroporation, in particular the positioning of the probe containing the syringe allowing tissue irrigation and the penetration of the selected substances into the dermis. The electrical discharge from the microcurrent simultaneously opens the plasma membrane of cells in many places, allowing molecules to enter.

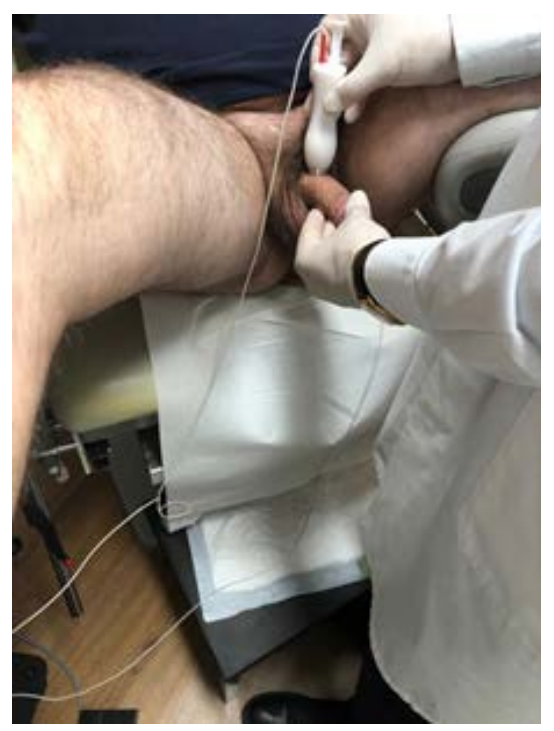

Figure 7. The syringe containing the substances to be injected is inserted into the electroporation probe itself connected to electrodes releasing a mini electric current the intensity of which is adapted for each patient. This painless and non-invasive method causes a reversible and momentary increase in tissue permeability.

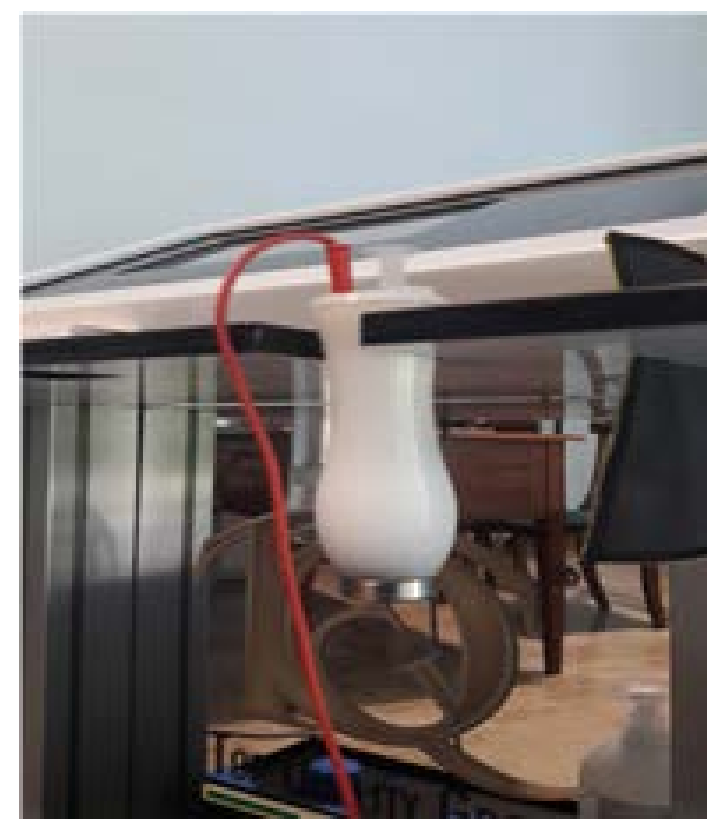

Figure 8. Treatment console for the application of the electroporation mini-current.

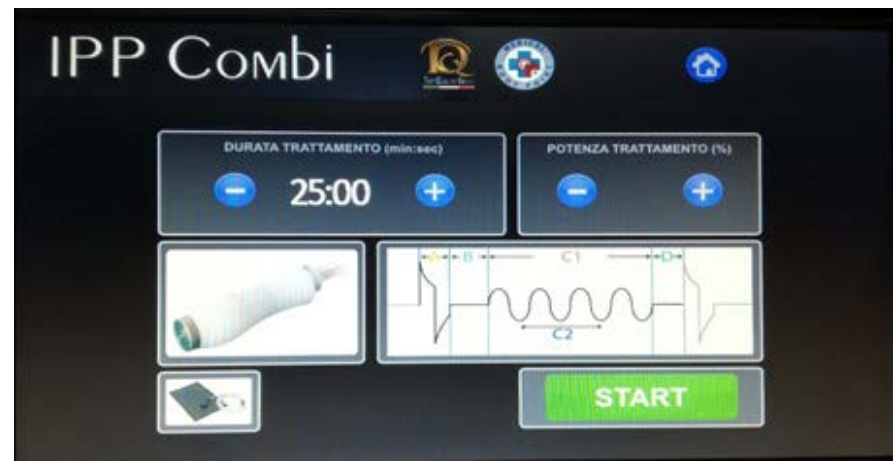

Table 2. Composition of the water-soluble solution used for electroporation. The components are diluted in $50 \mathrm{ml}$ of physiological solution.

\begin{tabular}{|l|l|l|l|}
\hline Substance & Effet & Dosage & Note \\
\hline Vérapamil & Calcium antagonist and vasodilator & $10 \mathrm{ml}$ & \\
\hline Hydrocortisone & $\begin{array}{l}\text { Anti-inflammatory by inhibition of phos- } \\
\text { pholipase A2 }\end{array}$ & $100 \mathrm{mg}$ & \\
\hline Collagénase & Anti-inflammatory and fibrinolysis & Ointment & Optional \\
\hline Vitamine E & Anti-oxidant & $59 \mathrm{ml}$ & \\
\hline
\end{tabular}

\section{In Practice}

The combination of PPI and electroporation greatly improves patient management and functional outcomes. We illustrate this with a classic case of a patient with typical Peyronie's disease with troublesome distal curvature and moderate erectile dysfunction. [Figure 9,10]

Figure 9. A 65-year-old married patient with Peyronie's disease in the form of a fibrous plaque causing a distal ventral curvature. The erectile dysfunction was moderate but the discomfort evident.

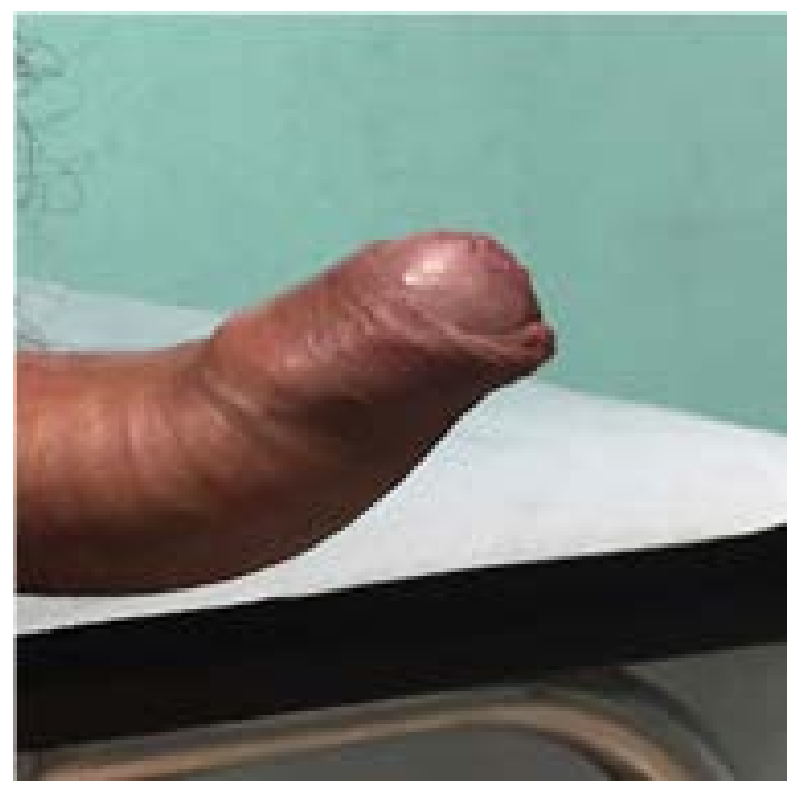


Figure 10. The treatment with hard mechanical waves (PPI) combined with electroporation at the rate of two sessions per week began 3 months after the appearance of the fibrous nodule and the curvature.

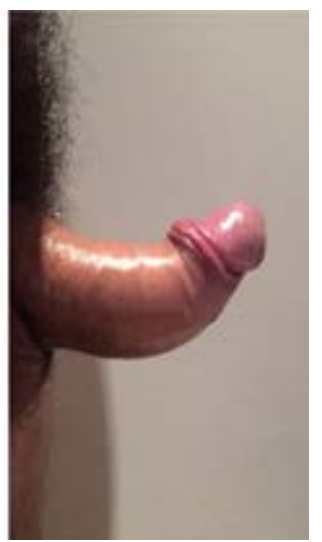

\section{CONCLUSION}

Peyronie's disease is a condition known for a long time but which has long been stigmatized as a malformation, misunderstood and generating shame and embarrassment on the part of patients. Doctors, whether specialized or not, have sometimes taken an approach of denial or trivialization due to a lack of understanding of the pathophysiology, as well as the evident ineffectiveness of many treatments offered.

Because of its multifactorial aspect and its association with certain metabolic diseases, the treatment for many years consisted of a simple observation, the prescription of vitamin E or other anti-inflammatory or vasodilator molecules.

Surgery remains the only radical treatment that can correct the organ, but at the cost of a sometimes heavy gesture and sometimes causing functional consequences.

We present here a new acquisition thanks to the application of linear shock waves, a promising and quite interesting element in the therapeutic arsenal. This technique, especially when coupled with the use of PDE-5 inhibitors and electroporation should make it possible to treat and improve not only punctually the fibrous nodule by improving the elasticity of the tissues concerned, but also of activate the circulation of the cavernous bodies of the penis thus improving erectile function allowing to give hope to many patients who are desperately seeking a solution to their problem.

\section{CONFLICTS OF INTEREST}

None.

\section{REFERENCES}

1. Jordan GHM. Peyronie's disease. In: Kavoussi LR ed. Campbell-Walsh Urology, Vol 1, $10^{\text {th }}$ ed, Philadelphia: Elsevier Saunders 2012; 792: 809.

2. Mulhall JP, Creech SD, Booriian SA. Subjective and Objective Analysis Of The Prevalence of Peyronie's Disease in A Population of Men Presenting For Prostate Cancer Screening. J Urol. 2004; 171: 2350-2353. doi: 10.1097/01.ju.0000127744.18878.f1

3. Mulhall JP, Schiff J, Guhring P. An Analysis of The Natural History of
Peyronie's Disease. J Urol. 2006; 175: 2115-2118. doi: 10.1016/S00225347(06)00270-9

4. Ralph DJ, Schwartz G, Moore W. The Genetic and Bacteriological Aspects of Peyronie's Disease. J Urol. 1997; 157: 291-294.

5. Haag SM, Hauck EW, Szardening-Kirchner C, Thorsten Diemer, Eun-Sook Cha, Wolfgang Weidner, et al. Alterations in the Transforming Growth Factor (TGF)-beta Pathway As A Potential Factor In The Pathogenesis of Peyronie's Disease. Eur Urol. 2007; 51: 255-261. doi: 10.1016/j.eururo.2006.05.002

6. Arafa M, Eid H, El-Badry A, et al. The Prevalence of Peyronie Disease in Diabetic Patients With Erectile Dysfunction. Int J Imp. 2007, 19: 213 7. doi: $10.1038 /$ sj.ijir.3901518

7. Bjekic MD, Vlajinac HD, Sipetic SB, Jelena M Marinkovic. Risk factors for Peyronie's Disease: A Case-Control Study. BJU Int. 2006; 97: 570-574. doi: 10.1111/j.1464-410X.2006.05969.x

8. Qian A, Meals RA, Rajfer J. Comparison of Gene Expression Profiles Between Peyronie's Disease and Dupuytren's Contracture. Urology. 2004; 64: 399-404. doi: 10.1016/j.urology.2004.04.006

9. Ohebshalom M, Mulhall J, Guhring P, MarilynParkerRN. Measurement of Penile Curvature in Peyronie's Disease Patients: Comparison of Three Methods. J Sex Med. 2007; 4: 199-203. doi: 10.1111/j.17436109.2006.00404.x

10. Karin S, Coyne MPH, Brooke MT, Smith. The Test-retest Reliability of the Peyronie's Disease Questionnaire. J Sex Med. 2015; 12: 543-548. doi: $10.1111 /$ jsm. 12769

11. Ralph D, Gonzalez-Cadavid N, Mirone V, et al. The Management of Peyronie's Disease: Evidence-Based 2010 guidelines. J Sex Med. 2010; 7: 2359-2374. doi: 10.1111/j.1743-6109.2010.01850.x

12. Martin DJ, Badwan K, Parker M, John P Mulhall. Transdermal Application of Verapamil Gel to The Penile Shaft Fails to Infiltrate The Tunica Albuginea. J Urol. 2002; 168: 2483-2485. doi: 10.1097/01. ju.0000031440.26763.da

13. Di Stasi SM, Giannantoni A, Stephen RL, et al. A Prospective, Randomized Study Using Transdermal Electromotive Administration of Verapamil And Dexamethasone For Peyronie's Disease. J Urol. 2004; 171: 1605-1608. doi: 10.1097/01.ju.0000116450.82816.2c

14. Hellstrom WJ, Kendirci M, Matern R, et al. Single-Blind, Multicenter, Placebo Controlled, Parallel Study to Assess The Safety and Efficacy of Intralesional Interferon Alpha-2b For Minimally Invasive Treatment for Peyronie's Disease. J Urol. 2006; 176: 394-398. doi: 10.1016/ s0022-5347(06)00517-9

15. Gelbard M, Goldstein I, Hellstrom WJ, et al. Clinical Efficacy, Safety and Tolerability of Collagenase Clostridium Histolyticum for The Treatment of Peyronie Disease in 2 Large Double-Blind, Randomized, Placebo Controlled Phase 3 Studies. J Urol. 2013; 190: 199-207. doi: 10.1016/j.juro.2013.01.087

16. Ajay N, Ralph A, Daniel J, et al. Peyronie's Disease: AUA guideline. J Urol. 2015; 194(3): 745-753. doi: 10.1016/j.juro.2015.05.098

17. Levine LA, Larsen SM. Surgery for Peyronie's Disease. Asian J Androl. 2013; 15: 27-34. doi: 10.1038/aja.2012.92

18. Greenfield JM, Lucas S, Levine LA. Factors Affecting The Loss of 
Length Associated with Tunica Albuginea Plication for Correction of Penile Curvature. J Urol. 2006; 175: 238-241. doi: 10.1016/S00225347(05)00063-7

19. Segal RL, Burnett AL. Surgical management for Peyronie's disease. World J Mens Health. 2013; 31: 1-11. doi: 10.5534/wjmh.2013.31.1.1

20. Kadioglu A, Akman T, Sanli O, Levent Gurkan, Murat Cakan, Mu- rat Celtik. Surgical Treatment of Peyronie's disease: A Critical Analysis. Eur Urol. 2006; 50: 235-248. doi: 10.1016/j.eururo.2006.04.030

21. Yafi FA, Sangkum P, McCaslin IR, Wayne J G Hellstrom. Strategies for Penile Prosthesis Placement in Peyronie's Disease and Corporal Fibrosis. Curr Urol Rep. 2015; 16: 21. doi: 10.1007/s11934-015-0491-0 